\title{
Platelet-Derived Microparticles in Patients with Colorectal Cancer
}

\author{
Violetta Dymicka-Piekarska ${ }^{1 *}$, Mariusz Gryko ${ }^{2}$, Alina Lipska ${ }^{3}$, Aleksandra Korniluk ${ }^{1}$, \\ Elżbieta Siergiejko ${ }^{1}$, Halina Kemona ${ }^{1}$ \\ ${ }^{1}$ Department of Clinical Laboratory Diagnostics, Medical University of Bialystok, Bialystok, Poland; ${ }^{2}$ Department of General and \\ Gastroenterological Surgery, Medical University of Bialystok, Bialystok, Poland; ${ }^{3}$ Regional Centre for Transfusion Medicine, Bialy- \\ stok, Poland. \\ Email: * piekarskav@yahoo.com
}

Received September $23^{\text {rd }}, 2012$; revised October $22^{\text {nd }}, 2012$; accepted October $31^{\text {st }}, 2012$

\begin{abstract}
Microparticles (MPs) are membrane vesicles released by platelets during activation or apoptosis. PMPs stimulate coagulation and cell adhesion to the vascular endothelium, both of which being important in the development of cancer progression. We investigated the correlation between microparticles and cancer advancement. The percentage of PMPs was measured by flow cytometry in 22 colorectal cancer patients and in 20 healthy subjects. The patients were divided into two groups according to the presence or lack of lymph node metastases. The percentage of PMPs was significantly higher in patients than in healthy subjects $(\mathrm{p}<0.001)$. We observed a positive correlation between PMPs and the presence of lymph node metastases $(r=0.63, p<0.003)$. This high correlation may suggest that PMPs are associated with lymph node involvement and cancer progression.
\end{abstract}

Keywords: Microparticles; Platelets; Colorectal Cancer

\section{Introduction}

Recent years have brought an increasing interest in the potential role of microparticles in the pathogenesis of various diseases. Microparticles (MPs), also known as microvesicles (MVs), are $0.1-1.0 \mu \mathrm{m}$ membrane vesicles, released from the surface of inflammatory cells, endothelial cells, blood platelets and megakaryocytes during their activation or apoptosis [1,2]. MP secretion by blood cells is a physiological function and there have been several reports suggesting that MPs play a biological function other than hemostasis; they can stimulate the proliferation of smooth muscle cells [3] and hematopoietic cells [4]. Their increased release is known to accompany many diseases, including cancers, sepsis, atherosclerosis, diabetes and severe injuries [5-9].

The role of circulating MPs in the initiation of the coagulation process is well known. The microparticles rich in phospholipids (microphosphatidyloserine-FS) and tissue factor (TF) are responsible for enhanced procoagulative activity and take part in the development of thromboembolic complications [10,11]. However, little is known about their role in neoplastic disease, although according to some authors, high levels of MPs correlate with cancer aggressiveness and poor prognosis $[4,11-13]$.

"Corresponding author.
According to Ardoin et al., MPs may represent a novel marker of disease activity [14].

Among the MPs released from the surface of various cells, platelet-derived microparticles (PMP) appear to predominate [15]. Blood platelets (PLT) are known to be actively involved in all stages of the metastatic cascade and angiogenesis, thus contributing to neoplastic growth and progression. PMPs express P-selectin glycoprotein ligand (PSGL-1), and contain chemokines (e.g. IL-1 $\beta$ ) and vascular endothelial growth factor (VEGF) that take part in cancer development [6]. In our study, attempts were undertaken to investigate the hypothesis whether the microparticles released from the activated PLTs mediate cancer dissemination. Thus, the study objective was to evaluate the percentage of PMPs, by using the flow cytometry method, with regard to the presence or lack of metastases in colorectal cancer patients.

The correlations observed between the percentage of PMPs and the presence of metastases may indirectly indicate their involvement in the dissemination of cancer cells and neoplastic progression.

\section{Material and Methods}

\subsection{Patients}

The study involved 22 patients with colorectal cancer 
(Adenocarcinoma) (10 women and 12 men, aged 53 - 72 years) and 20 healthy subjects as the control group (9 woman and 11 men, aged $49-67$ years). The patients were treated in 2nd Department of General and Gastroenterological Surgery, University Hospital in Bialystok. The diagnosis of colorectal cancer (CRC) was based on clinical symptoms and imaging investigations (ultrasonography, radiology and CT), as well as endoscopy (colonoscopy).

The patients were divided into 2 groups according to the presence or lack of lymph node metastases: group A-11 patients without metastases (T1-4N0M0) and group B-11 patients with lymph node involvement $(\mathrm{T} \times \mathrm{N}+\mathrm{M} 0)$.

The colorectal cancer patients and control subjects qualified for our study did not have any symptoms that could affect the immune response. Those who had taken aspirin or other drugs affecting platelet function in the preceding week were excluded from the study. All patients were qualified for surgery and received no anticancer treatment. According to the Guidelines for Good Clinical Practice (R-I-002/56/2010), all the patients gave consent for participation in the study.

\subsection{Methods}

PMP analysis. The percentage of PMPs was analyzed using commercially available fluorescent microbeads of defined concentration as a reference standard.

Samples of venous blood $(2.7 \mathrm{~mL})$ were drawn into Vacutainer tubes containing EDTA (Sarstedt) and tested immediately after collection. To receive PRP (platelet-rich plasma), the sample centrifuged for $15 \mathrm{~min}$ at $1550 \times$ g. $20 \mu \mathrm{l}$ of plasma was incubated in dark with $5 \mu \mathrm{l}$ platelet-specific antibody (CD 61 PerCP) (Becton Dickinson). After 10-minute incubation the sample was diluted in $500 \mu \mathrm{l}$ of $0.5 \%$ paraformaldehyde/phosphatebuffered saline (PBS) and $20 \mu \mathrm{l}$ of Flow Count Fluorospheres (Microbead NIST Traceable Particle Size Standard, Polysciences Inc., USA) were added. The sample was analyzed within $30 \mathrm{~min}$. by a FACS Calibur Cytometer (Becton Dickinson). Only cells and particles positive for $\mathrm{CD} 61$ were gated to separate the platelets and PMPs. The PMP gate was defined using $\leq 1 \mu \mathrm{l}$ diameter beads. A total of $10,000 \mathrm{CD} 61$ positive cells were counted form each sample and analyzed.

Platelet count. In the same blood samples, platelet count also underwent automatic assessment on a hematological analyzer Advia 2120 (Siemens).

\subsection{Statistical Analysis}

Study results were statistically analyzed based on the STATISTICA 8.0 PL. The hypothesis of normal distribution was verified with Kolmogorov test. Differences between the groups were evaluated with Student's $t$ test for matched pairs. The Spearman's rank correlation coefficient was also calculated. p-values less than 0.05 were considered significant. Results are presented as mean values \pm standard deviation (SD).

\section{Results}

Table 1 shows the means and SD of the percentage of PMP and PLT count in patients with CRC and in control group. The percentage of PMPs was four times higher in colorectal cancer patients than in healthy subjects $(p<$ 0.001 ) and showed a growing tendency with the clinical stage of the cancer. We found a statistically significant increase in the percentage of PMPs in patients with local lymph node involvement (group B) as compared to patients with a tumor restricted to the colonic wall (group A) $(\mathrm{p}<0.05)$.

Simultaneously, we noted a statistically significantly higher platelet count in the study patients as compared to the control $(p<0.009)$. Contrary to PMP, the PLT count in the metastasis-free group (group A) was significantly higher than in the group with lymph node involvement (group B). In our opinion, platelet count reduction was accompanied by an increase in their activation, which was manifested by substantial release and increased percentage of PMPs observed in patients with more advanced colorectal cancer.

Additionally, in metastatic patients (group B) we observed a positive correlation between the percentage of PMPs and the presence of lymph node metastases $(r=$ $0.63 ; \mathrm{p}<0.003)$. Statistical analysis did not reveal a correlation between platelet derived microparticles and PLT $(\mathrm{r}=-0.21 ; \mathrm{p}=0.408)$.

\section{Discussion}

PMP involvement in the development of cancer is an interesting and not entirely elucidated phenomenon. Our results seem to confirm their participation in this process. As we know, PMPs possess numerous adhesion molecules on their surface, e.g. P-selectin or CD $40 \mathrm{~L}$, by

Table 1. Percentage of PMP and platelet count in colorectal cancer (CRC) according to clinical advancement and in control group.

\begin{tabular}{ccc}
\hline & $\begin{array}{c}\text { PMP }(\%) \\
(\text { mean } \pm \text { S.E. })\end{array}$ & $\begin{array}{c}\text { PLT }\left(\times 10^{3} / \mu \mathrm{L}\right) \\
(\text { mean } \pm \text { S.E. })\end{array}$ \\
\hline CRC patients $(\mathrm{n}=22)$ & $10.12 \pm 2.42$ & $266.21 \pm 92.36$ \\
Control group $(\mathrm{n}=20)$ & $2.46 \pm 0.67$ & $214.40 \pm 13.17$ \\
$\mathrm{p}$ & 0.001 & 0.009 \\
Group A $(\mathrm{n}=11)$ & $8.99 \pm 1.44$ & $289.34 \pm 81.12$ \\
Group B $(\mathrm{n}=11)$ & $12.31 \pm 1.26$ & $249.29 \pm 89.84$ \\
$\mathrm{p}$ & 0.05 & $\mathrm{NS}$ \\
\hline
\end{tabular}

Group A-patients without metastases; Group B - patients with lymph node involvement. 
means of which they are bound to hematopoietic cells, inflammatory cells and cancer cells, thus taking part in intercellular signal transmission and modulating the functions of these cells [4]. Due to their small sizes, microparticles easily move in the circulatory system, also at the sites that are distant from a primary lesion and in microcirculation. Thus, they can promote tumor spread and metastasizing.

Our study confirmed a substantial release of microparticles from active platelets in patients with colorectal cancer. The percentage of PMPs was repeatedly higher in comparison with healthy subjects. Moreover, we observed much higher PMP count in patients with lymph node metastases (group B) than in patients with cancer limited to the colonic wall (group A). Additionally, in group B we found a positive correlation between PMPs and the presence of metastases, which could indicate PMP involvement in the formation of metastases and cancer progression.

At the same time, statistical analysis did not reveal a correlation between platelet-derived MPs and PLT count, which may indirectly prove that also other cells, such as megakaryocytes, could be the source of CD $61+$ PMPs. Another possible explanation of the lack of correlation between PMPs and PLTs is a small number of patients in the study group. Interestingly, other authors more frequently showed a relationship between PMPs and PLT activation markers (CD 62P and CD 63), indicating their usefulness as platelet activation indices [7].

Enhanced metastatic and angiogenic potential of PMPs in lung cancer has been demonstrated by JanowskaWieczorek et al. [14]. PMPs increase adhesion of cancer cells to the endothelium by transmitting numerous integrins characteristic of PLT from which they are derived and taken to the metastatic site. It thus seems that due to greater adhesion ability and supply of metalloproteinases, PMPs promote migration of cancer cells beyond the vascular lumen [6]. Then, through the transmission and release of factors involved in tumor vascularization, i.e. VEGF and HGF, PMPs participate in this essential aspect of tumor invasion. They also promote proliferation and migration of endothelial cells, which also facilitates this process $[11,12]$.

Like PLTs, also PMPs are engaged in the initiation and enhancement of the inflammatory process within a neoplastic lesion. On one hand, mediated by surface receptors, they get involved in intercellular interactions with inflammatory cells; on the other, they release many relevant cytokines, e.g. RANTES, IL-1 $\beta,[6,13]$, which may also affect tumor growth and progression.

However, as revealed by our brief study, the percentage of PMPs released from the surface of active platelets in colorectal cancer patients was significantly higher than in the group of healthy subjects, and at the same time much higher in patients with local lymph node involvement. Moreover, PMPs were found to show a statistically significant correlation with the presence of metastases, which may indirectly prove that they are involved in metastasis formation. However, the role of PMPs in tumor progression requires further research.

\section{REFERENCES}

[1] M. D. Shah, A. L. Bergeron, J. F. Dong, et al., "Flow Cytometric Measurement of Microparticles: Pitfalls and Protocol Modifications," Platelets, Vol. 19, No. 5, 2008, pp. 365-372. doi:10.1080/09537100802054107

[2] A. R. Beaudoin and G. Grondin, "Shedding of Vesicular Material from the Cell Surface of Eucaryotic Cells: Different Cellular Phenomena," Biochimica et Biophysica Acta, Vol. 1071, No. 3, 1991, pp. 203-219. doi:10.1016/0304-4157(91)90014-N

[3] A. Weber, H. O. Koppen and K. Schror, "Platelet-Derived Micropartocles Stimulate Coronary Artery Smooth Muscle Cell Mitogenesis by a PDGF-Independent Mechanism," Thrombosis Research, Vol. 98, No. 5, 2000, pp. 461-466. doi:10.1016/S0049-3848(00)00192-4

[4] M. Baj-Krzyworzeka, M. Majka, D. Pratico, et al., "Platelet-Derived Microparticles Stimulate Proliferation, Survival, Adhesion, and Hemotaxis of Hematopoietic Cells," Experimental Hematology, Vol. 30, No. 5, 2002, pp. 450459. doi:10.1016/S0301-472X(02)00791-9

[5] J. Simak and M. P. Gelderman, "Cell Membrane Microparticles in Blood and Blood Products: Potentially Pathogenic Agents and Diagnostic Markers," Transfusion Medicine Reviews, Vol. 20, No. 1, 2006, pp. 1-26. doi:10.1016/i.tmrv.2005.08.001

[6] J. Rak, "Microparticles in Cancer," Seminars in Thrombosis and Hemostasis, Vol. 36, No. 8, 2010, pp. 888-906. doi: $10.1055 / \mathrm{s}-0030-1267043$

[7] N. Ogata, M. Imaizumi, S. Nomura, et al., "Increased Levels of Platelet-Derived Microparticles in Patients With Diabetic Retinopathy," Diabetes Research and Clinical Practice, Vol. 68, No. 3, 2005, pp. 193-201. doi:10.1016/j.diabres.2004.10.010

[8] B. Toth, S. Liebhardt, K. Steinig, et al., "Platelet-Derived Microparticles and Coagulation Activation in Breast Cancer Patients," Journal of Thrombosis and Haemostasis, Vol. 100, No. 4, 2008, pp. 663-669.

[9] M. C. Martinez, A. Tesse, F. Zobairi, et al., "Shed Membrane Microparticles from Circulating and Vascular Cells in Regulation Vascular Function," American Journal of Physiology-Heart and Circulatory Physiology, Vol. 288, No. 3, 2005, pp. 1004-1009. doi:10.1152/ajpheart.00842.2004

[10] D. Castellana, F. Toti and J. M. Freyssinet, "Membrane Microvesicles: Macromessengers in Cancer Disease and Progression," Thrombosis Research, Vol. 125, No. 2, 2010, pp. S84-S88. doi:10.1016/S0049-3848(10)70021-9

[11] D. Varon and E. Shai, "Role of Platelet-Derived Microparticles in Angiogenesis and Tumor Progression," Discovery Medicine, Vol. 8, No. 43, 2009, pp. 237-241. 
[12] H. K. Kim, K. S. Song, J. H. Chung, et al., "Platelet Microparticles Induce Angiogenesis in Vitro," British Journal of Haematology, Vol. 124, No. 3, 2004, pp. 376-384. doi:10.1046/j.1365-2141.2003.04773.x

[13] S. P. Ardoin, J. C. Shanahan and D. S. Pisetsky, "The Role of Microparticles in Inflammation and Thrombosis," Scandinavian Journal of Immunology, Vol. 66, No. 1, 2007, pp. 159-165.

doi:10.1111/j.1365-3083.2007.01984.x
[14] A. Janowska-Wieczorek, M. Wysoczynski, J. Kijowski, et al., "Microvesicles Derived from Activated Platelets Induce Metastasis and Angiogenesis in Lung Cancer," International Journal of Cancer, Vol. 113, No. 5, 2005, pp. 752-760. doi:10.1002/ijc. 20657

[15] R. Flaumenhaft, A. T. Mairuhu and J. E. Italiano, "Platelet and Megakaryocyte-Derived Microparticles," Seminars in Thrombosis and Hemostasis, Vol. 36, No. 8, 2010, pp. 881-887. doi:10.1055/s-0030-1267042 